\title{
Synergistic anti-proliferative effects of gambogic acid with docetaxel in gastrointestinal cancer cell lines
}

\author{
Zhengyun Zou', Li Xie', Jia Wei', Lixia Yu', Xiaoping Qian'1, Junhao Chen², Tingting Wang ${ }^{3}$ and Baorui Liu*
}

\begin{abstract}
Summary
Background: Gambogic acid has a marked anti-tumor effect for gastric and colorectal cancers in vitro and in vivo. However, recent investigations on gambogic acid have focused mainly on mono-drug therapy, and its potential role in cancer therapy has not been comprehensively illustrated. This study aimed to assess the interaction between gambogic acid and docetaxel on human gastrointestinal cancer cells and to investigate the mechanism of gambogic acid plus docetaxel treatment-induced apoptotic cell death.

Methods: MTT assay was used to determine $\mathrm{IC}_{50}$ values in BGC-823, MKN-28, LOVO and SW-116 cells after gambogic acid and docetaxel administration. Median effect analysis was applied for determination of synergism and antagonism. Synergistic interaction between gambogic acid and docetaxel was evaluated using the combination index (CI) method. Furthermore, cellular apoptosis was analyzed by Annexin-V and propidium iodide (PI) double staining. Additionally, mRNA expression of drug-associated genes, i.e., $\beta$-tublin III and tau, and the apoptosis-related gene survivin, were measured by quantitative reverse transcription polymerase chain reaction (qRT-PCR).
\end{abstract}

Results: Gambogic acid provided a synergistic effect on the cytotoxicity induced by docetaxel in all four cell lines. The combined application of gambogic acid and docetaxel enhanced apoptosis in gastrointestinal cancer cells. Moreover, gambogic acid markedly decreased the mRNA expression of docetaxel-related genes, including $\beta$-tubulin III, tau and survivin, in BGC-823 cells.

Conclusions: Gambogic acid plus docetaxel produced a synergistic anti-tumor effect in gastrointestinal cancer cells, suggesting that the drug combination may offer a novel treatment option for patients with gastric and colorectal cancers.

\section{Background}

Despite a decline in incidence, gastric cancer remains one of the leading causes of cancer-related deaths worldwide [1]. Colorectal cancer is the third most common cancer worldwide, with an incidence of approximately one million cases and 500,000 mortalities annually [2,3]. Despite recent advances in chemotherapeutic treatment of gastric cancer, no single agent or combination regimen has been accepted as a standard therapy [4]. For colorectal cancer, capecitabine monotherapy or combination chemotherapies such as FOLFOX (infusional 5-fluorouracil/leucovorin with oxaliplatin)

\footnotetext{
* Correspondence: baoruiliu@nju.edu.cn

${ }^{1}$ The Comprehensive Cancer Center of Drum Tower Hospital, Medical School of Nanjing University \& Clinical Cancer Institute of Nanjing University, Nanjing 210008, China

Full list of author information is available at the end of the article
}

and FOLFIRI (infusional 5-fluorouracil/leucovorin with irinotecan) are considered standard regimens. However, the positive response rate is still lower than $40 \%[5,6]$. Hence, better systemic therapies are needed to decrease side effects and improve the clinical outcomes of patients with gastric and colorectal cancer.

Docetaxel (Doc), a widely used taxane for the treatment of a variety of cancers, shares a similar mechanism of action with paclitaxel [7]. Emerging lines of evidence indicate that Doc is quite active against several human malignancies, including gastric cancer [8,9]. Nevertheless, a poor prognosis has been observed after Doc monotherapy. A previous report demonstrated that administration of $100 \mathrm{mg} / \mathrm{m}^{2}$ Doc to patients with adenocarcinoma of the upper gastrointestinal tract, previously untreated with cytotoxic chemotherapy, yielded a $17 \%$ response rate and only 2.8 months of

\section{Biomed Central}

(C) 2012 Zou et al; BioMed Central Ltd. This is an Open Access article distributed under the terms of the Creative Commons Attribution License (http://creativecommons.org/licenses/by/2.0), which permits unrestricted use, distribution, and reproduction in any medium, provided the original work is properly cited. 
median time of progression (TTP)[9]. It has been reported that Doc plus cisplatin induces an overall response rate of $37.2 \%$ in patients with advanced gastric cancer, [10] suggesting that Doc-containing combination therapy might be useful in the management of gastric cancer.

Our previous study demonstrated that gambogic acid (GA), the primary active component of gamboge, reversed Doc resistance in BGC-823/Doc gastric cancer cells [11]. Moreover, GA alone or in combination with Doc significantly down-regulates the mRNA expression of survivin in gastric cancer cells, [11] implying that GA may promote the anti-tumor effect of Doc through promotion of apoptotic cell death. However, the synergistic anti-tumor effect of GA and Doc in gastrointestinal cancer cells has not yet been clearly illustrated.

In the present study, two gastric cancer cell lines, MKN-28 and BGC-823, and two colorectal cancer cell lines, LOVO and SW-116, were used to examine the effects of GA and Doc mono-therapy or combination therapy. Our results indicated that administration of GA plus Doc enhanced apoptotic cell death in gastrointestinal cancer cells compared to a single drug application, suggesting that this drug combination may provide a novel and beneficial treatment option for patients with gastric and colorectal cancers.

\section{Methods \\ Reagents}

Gambogic acid (GA, molecular formula $\mathrm{C}_{38} \mathrm{H}_{44} \mathrm{O}_{8}$ ) was provided by Jiangsu Kangyuan Pharmaceutical Co., Ltd. (Jiangsu, China). Docetaxel (Doc) was obtained from Jiangsu Hengrui Pharmaceutical Co., Ltd. (Jiangsu, China). GA and Doc were prepared in complete culture medium immediately prior to use. RPMI 1640 medium was purchased from GIBCO BRL (Grand Island, NY, USA). Fetal bovine serum was obtained from Lanzhou National Hyclone Bio-engineering Co., Ltd. (Lanzhou, Gansu, China). MTT (3-[4, 5-dimethylthiazol-2-yl]-2, 5-diphenyltetrazolium bromide) was purchased from Sigma Chemical Co. (St. Louis, MO, USA). Dimethyl sulfoxide (DMSO) was provided by Shanghai Ling-feng Chemical Reagents Co. (Shanghai, China). All other chemicals used in this study were of the highest purity available.

\section{Cell culture}

Human cancer cell lines, specifically the gastric cancer cell lines MKN-28 and BGC-823 and the colorectal cancer cell lines LOVO and SW-116, were obtained from Shanghai Institute of Cell Biology (Shanghai, China). Cells were maintained in RPMI 1640 medium supplemented with $10 \%$ fetal bovine serum. Cultures were incubated at $37^{\circ} \mathrm{C}$ in a humidified atmosphere of $5 \% \mathrm{CO}_{2}$.

\section{Evaluation of cytotoxicity}

Cytotoxicity was measured using a MTT assay. Briefly, tumor cells in log-phase were trypsinized and seeded at a density of $2 \times 10^{3}$ cells per well onto 96-well plates. After $24 \mathrm{~h}$, cells were treated with GA, Doc, or GA plus Doc at different concentrations. MTT (1/10 volume) was added to each well after 48-h drug treatments, and plates were further incubated at $37^{\circ} \mathrm{C}$ for another $4 \mathrm{~h}$. Formazan crystals formed were dissolved in DMSO. Absorbance (OD) was determined with a multiwell spectrophotometer (BioTek, Winooski, VT, USA) at $570 \mathrm{~nm}$. Absorbance values were expressed as percentages relative to untreated controls. The $\mathrm{IC}_{50}$ was defined as the concentration required for $50 \%$ inhibition of cell growth. Each condition was tested in quintuplicate, and at least three independent experiments were performed. The inhibition rate was calculated according to the following equation: Inhibition rate (IR) $(\%)=[$ (average OD value in the control group - average OD value in the treatment group) / average OD value in the control group] $\times 100 \%$.

\section{Determination of synergism and antagonism}

Synergism or antagonism after drug treatments was quantitated with the median-effect principle, using the combination index $(\mathrm{CI})$ method [12]. The CI is defined by the following equation: $\mathrm{CI}=[(\mathrm{D}) 1 /(\mathrm{Dx}) 1]+[(\mathrm{D}) 2 /$ $(\mathrm{Dx}) 2]+[\alpha(\mathrm{D}) 1(\mathrm{D}) 2 /(\mathrm{Dx}) 1(\mathrm{Dx}) 2]$, where $(\mathrm{Dx}) 1$ and $(\mathrm{Dx})$ 2 are the concentrations for D1 (GA) and D2 (Doc) that give $\mathrm{x} \%$ inhibition, whereas (D) 1 and (D) 2 in the numerators are the concentrations of the two drugs that produce an identical level of effect in combination. The parameter $\alpha=0$ when the drugs are mutually exclusive (i.e., with similar modes of action), while $\alpha=1$ if they are mutually non-exclusive (i.e., with independent modes of action). CI values $>1$ indicate antagonism, CI values $<1$ indicate synergy, and $\mathrm{CI}$ values $=1$ indicate additivity. Each $\mathrm{CI}$ ratio represented here is the mean value derived from at least three independent experiments.

\section{Assessment of apoptotic and necrotic cell death}

Apoptotic or necrotic cell death was evaluated using an annexin V-fluorescein isothiocyanate (FITC) Apoptosis Detection Kit (BioVision, Mountain View, CA, USA) according to the manufacturer's instructions. MNK-28 cells were treated with $0.25 \mu \mathrm{M}$ GA and $0.625 \mu \mathrm{M}$ Doc; BGC-823 cells were treated with $2.5 \mu \mathrm{M}$ GA and $6.25 \mu \mathrm{M}$ Doc; LOVO and SW-116 cells were treated with $1 \mu \mathrm{M}$ GA and $2.5 \mu \mathrm{M}$ Doc. After $48 \mathrm{~h}$ of drug treatment, cells were harvested and resuspended in $500 \mu \mathrm{L}$ of binding buffer. Annexin V-FITC and propidium iodide (PI) were then added. Percentages of apoptotic or necrotic cells were analyzed with a FACScan flow cytometer (Becton Dickinson, Sunnyvale, CA, USA). The cells in the annexin $\mathrm{V}^{+}$and $\mathrm{PI}^{-}$fraction were identified as early 

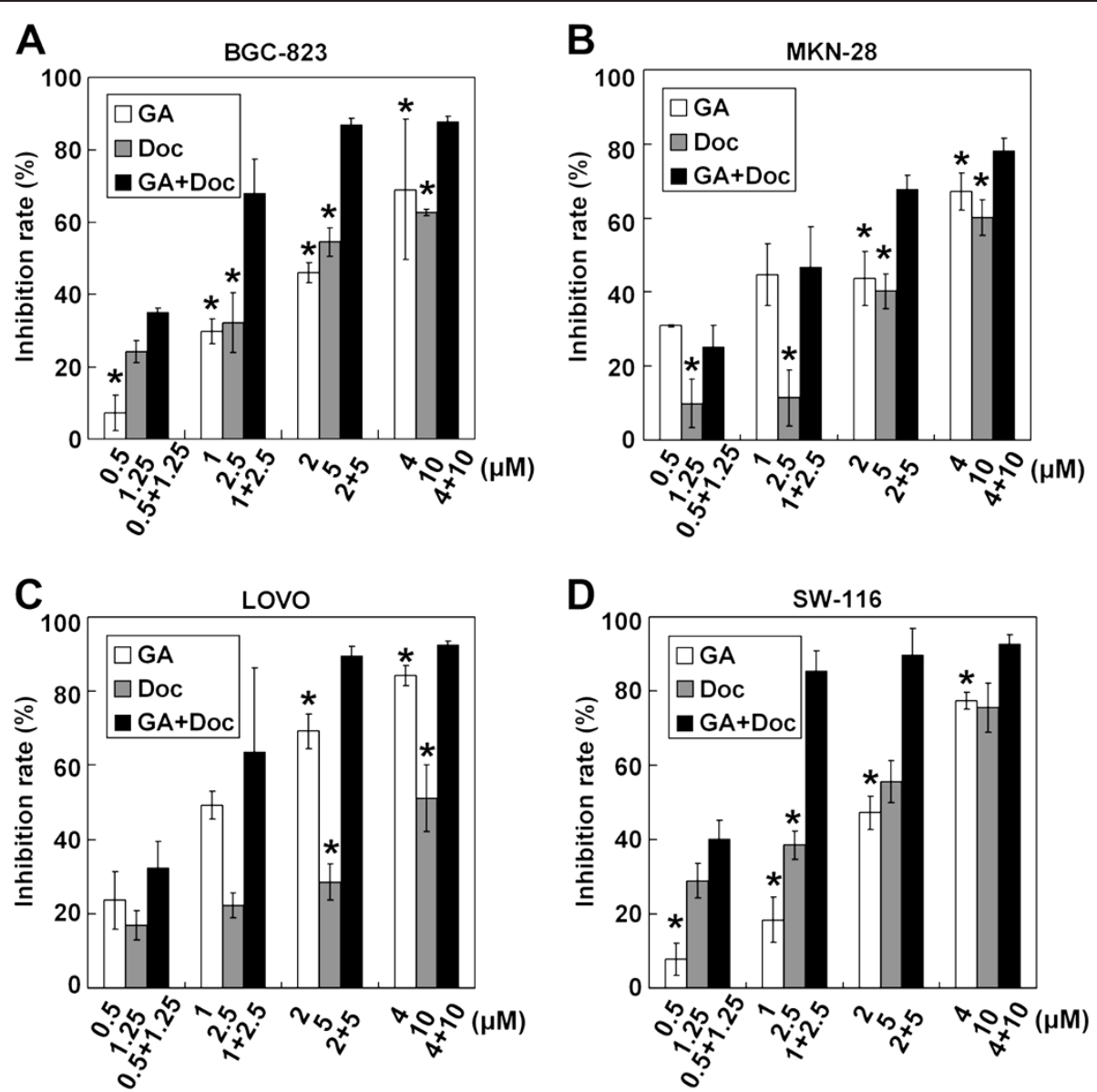

Figure 1 Cytotoxic effects of GA and Doc treatment in cancer cells. After $48 \mathrm{~h}$ incubation with GA, Doc or GA plus Doc at the indicated concentrations, cytotoxicity was determined by the MTT assay. Inhibition rates (\%) in BGC-823 (A), MKN-28 (B), LOVO (C) and SW-116 (D) cells were calculated as described in Methods. ${ }^{*} P<0.05$ compared with GA plus Doc.

apoptotic cells, while those in the annexin $\mathrm{V}^{+}$and $\mathrm{PI}^{+}$ fraction represented late apoptotic cells or necrotic cells.

\section{Quantitative reverse transcription polymerase chain reaction (qRT-PCR)}

Total RNA was extracted from BGC-823 cells after $48 \mathrm{~h}$ incubation with or without GA. cDNA was synthesized using random primers and Primescript reverse transcriptase (Takara; Shiga, Japan). qPCR reactions for indicated genes were carried out using SYBR green qPCR kit (Takara; Shiga, Japan) by a fluorescent temperature cycler (Mx3000P Real Time PCR System; Stratagene; Santa Clara, CA, USA). Sequences of primers (forward and reverse, respectively) were as follows: $\beta$-tubulin III: 5'-AGCAAGAACAGCAGCTACTTCGT-3' and 5'-GATGAAGGTGGAGGACATCTTGA-3'; tau: 5'- GATTGG GTCCCTGGACAATA-3' and 5'-GTGGTCTGTCTTG GCTTTGG-3'; survivin: 5'-ATTCGTCCGGTTGCGCT TTCC-3' and 5'-CACGGCGCACTTTCTTCGCAG-3'; $\beta$-actin: 5'-GCGAGAAGATGACCCAGATC-3' and 5'-
GGATAGCAACGCCTGGATAG-3'. Cycling conditions were as follows: denaturation $\left(95^{\circ} \mathrm{C}\right.$ for $\left.10 \mathrm{sec}\right)$, amplification and quantitation repeated for 45 cycles $\left(95^{\circ} \mathrm{C}\right.$ for $5 \mathrm{~s}, 60^{\circ} \mathrm{C}$ for $20 \mathrm{~s}$, with a single fluorescence measurement); a melting curve program $\left(95^{\circ} \mathrm{C}\right.$ for $10 \mathrm{~s}, 55-95^{\circ} \mathrm{C}$ with a heating rate of $0.1^{\circ} \mathrm{C} / \mathrm{s}$ and continuous fluorescence measurement). Relative gene expression was quantified according to the comparative $\mathrm{Ct}$ method using $\beta$-actin as an internal standard and cells without GA treatment as calibrators. Gene expression was

Table $1 \mathrm{IC}_{50}$ value ( $\left.\mu \mathrm{M}\right)$ of different cells after $48 \mathrm{~h}$ of $\mathrm{GA}$ or Doc treatment

\begin{tabular}{ccc}
\hline Cell line & GA & Doc \\
\hline BGC-823 & $2.33 \pm 0.05$ & $4.93 \pm 0.57$ \\
MKN-28 & $1.66 \pm 0.11$ & $8.63 \pm 1.90$ \\
LOVO & $1.21 \pm 0.11$ & $4.49 \pm 0.74$ \\
SW-116 & $2.14 \pm 0.33$ & $1.77 \pm 0.43$ \\
\hline
\end{tabular}




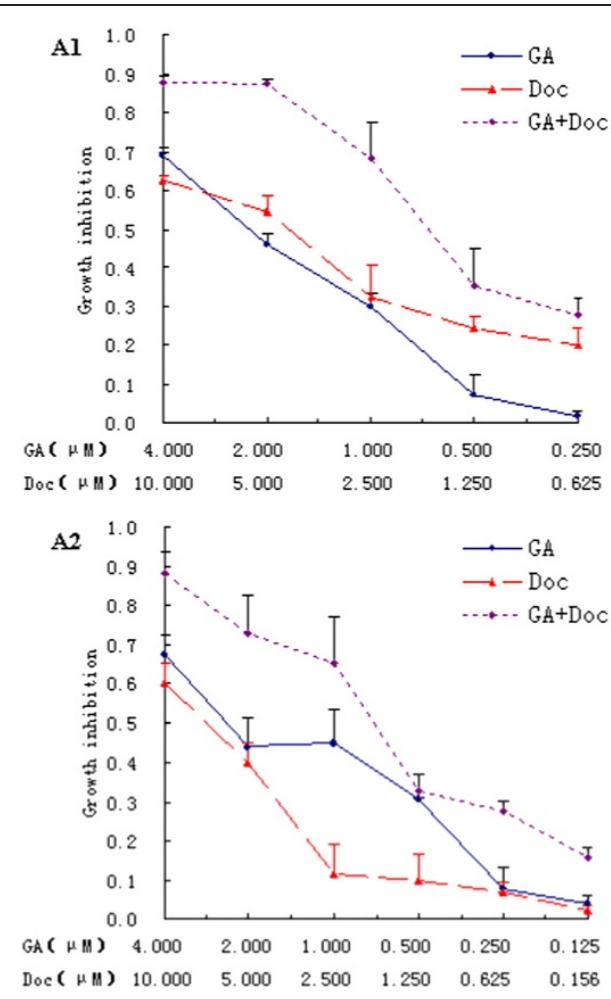

B1

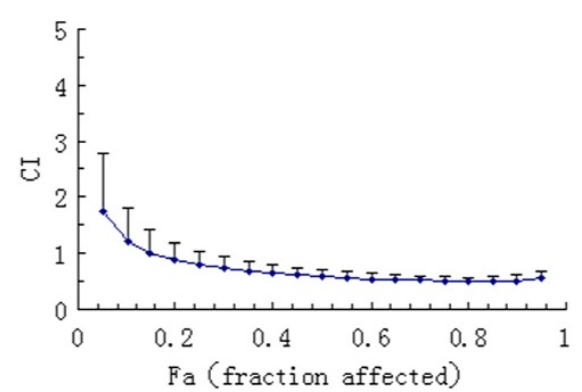

B2
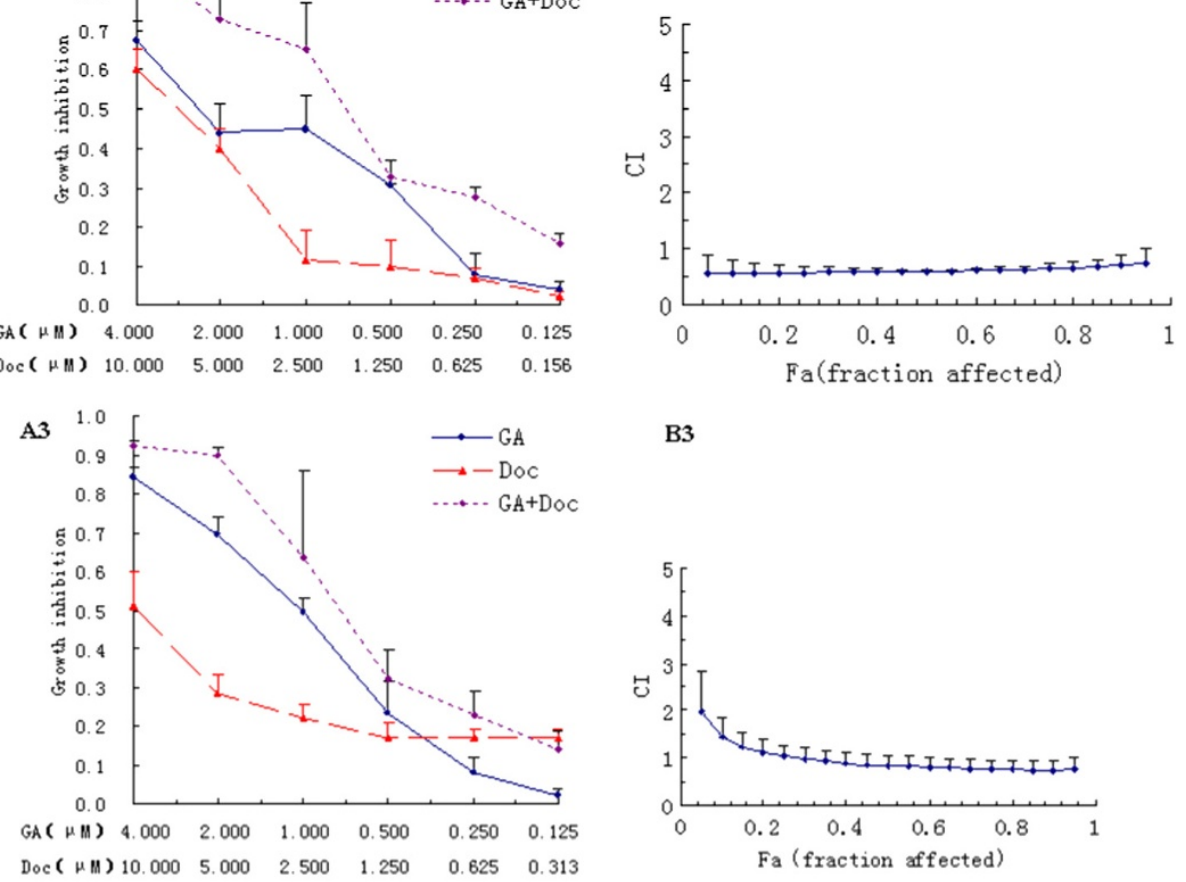

B3
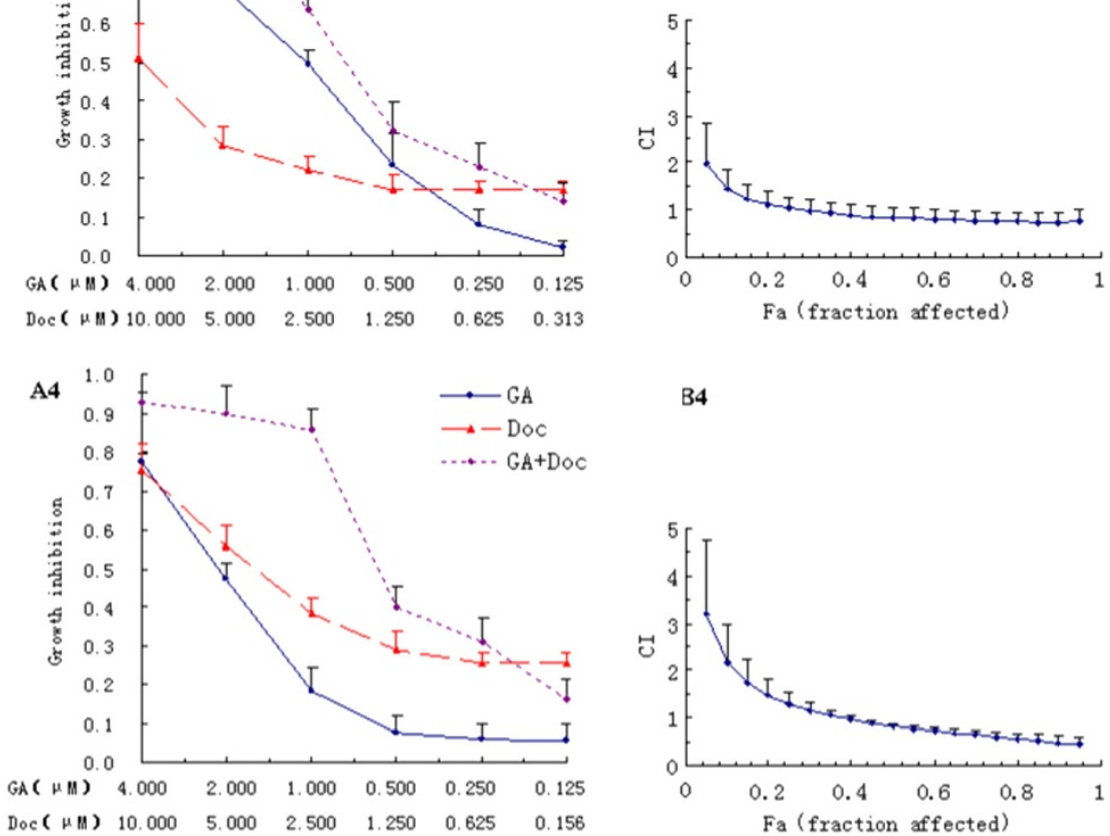

B4

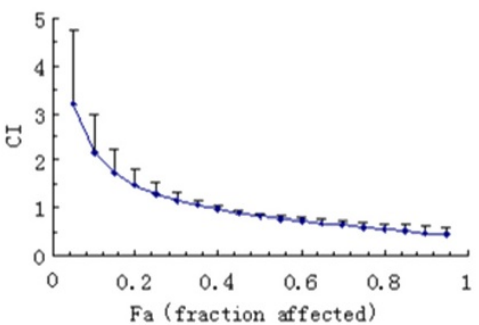

Figure 2 Synergistic effects of GA and Doc on gastrointestinal cancer cells. (A1-4): Dose-response curves for BGC-823 (A1), MKN-28 (A2), LOVO (A3) and SW116 (A4) cells. (B1-4): Cl values at different levels of growth inhibitory effect (the fraction of cells affected, Fa) for BGC-823 (B1), MKN28 (B2), LOVO (B3) and SW116 (B4) cells. 
Table 2 Summary of $\mathrm{Cl}$ values at $20 \%, 40 \%, 60 \%$ and $80 \%$ fraction affected

\begin{tabular}{lllll}
\hline \multicolumn{5}{c}{ Cl (means \pm SD) } \\
\hline Cell line & $20 \%$ & $40 \%$ & $60 \%$ & $80 \%$ \\
\hline BGC-823 & $0.87 \pm 0.31$ & $0.64 \pm 0.15$ & $0.54 \pm 0.09$ & $0.50 \pm 0.07$ \\
MKN-28 & $0.57 \pm 0.14$ & $0.59 \pm 0.06$ & $0.61 \pm 0.03$ & $0.65 \pm 0.11$ \\
LOVO & $1.11 \pm 0.26$ & $0.90 \pm 0.23$ & $0.80 \pm 0.21$ & $0.76 \pm 0.18$ \\
SW-116 & $1.47 \pm 0.34$ & $0.97 \pm 0.07$ & $0.73 \pm 0.08$ & $0.56 \pm 0.14$ \\
\hline
\end{tabular}

analyzed with the Stratagene analysis software and quantified by the $2^{-\Delta \Delta C t}$ method.

\section{Statistical analysis}

Data were analyzed by using SPSS 15.0 software and were expressed as means \pm SD. Statistical analysis was performed using the Student's $t$-test. Values of $P<0.05$ were considered to be statistically significant.

\section{Results}

Cytotoxic effects of GA and Doc treatment on cancer cells The cytotoxic effects of GA and Doc on cancer cells were first evaluated by using the MTT assay. As expected, GA or Doc alone dose-dependently increased cytotoxicity in the gastric cancer cell lines MKN-28 and BGC-823 and the colorectal cancer cell lines LOVO and SW-116 (Figure 1). Combined application of GA and Doc markedly enhanced the cytotoxic effects in each of the cell lines as compared with single drug administration $(P<0.05)$. Table 1 shows the half-maximal inhibitory concentration $\left(\mathrm{IC}_{50}\right)$ values for four cancer cells lines exposed to GA or Doc. It should be noted that the lowest $\mathrm{IC}_{50}$ for $\mathrm{GA}$ or Doc treatment was detected in LOVO $(1.21 \pm 0.11 \mu \mathrm{M})$ and SW-116 $(1.77 \pm 0.43 \mu \mathrm{M})$ cells, respectively. We then used fixed-ratio concentrations including each drug $\mathrm{IC}_{50}$ dose for the remaining experiments.

\section{Synergistic effects of GA and Doc on gastrointestinal cancer cells}

To investigate whether GA could enhance the antitumor effects of Doc, which is widely used in cancer chemotherapy, the effects of each drug (administered alone or in combination) were examined. The concentration ratio for the combined application of GA and Doc was set as 1: 2.5, which is approximately the same as the ratio of the $\mathrm{IC}_{50}$ values for each drug. Figure 2 shows the dose-response curves and CI values at different levels of growth inhibition effect (fraction affected, Fa) when GA and Doc were administrated alone or in combination to gastrointestinal cancer cells. In the gastric cancer cell lines MKN-28 and BGC-823 and the colorectal cancer cell lines LOVO and SW-116, the combination treatment provided an efficient anti-tumor response compared with single drug application, as revealed by the dose-response curves (Figure 2A1-4).

$\mathrm{CI}$ values at different levels of $\mathrm{Fa}$ demonstrated that when $\mathrm{Fa}>0.15$ for BGC-823 cells, $>0.25$ for LOVO cells, and $>0.40$ for SW-116 cells, the CI values were $<1$, and the combination treatment with GA and Doc could induce a synergistic, anti-proliferative effect (Figure 2B1-4). The CI value was $<1$ across almost the entire dose-inhibition range when GA was combined with Doc in MKN-28 cells, indicating a more

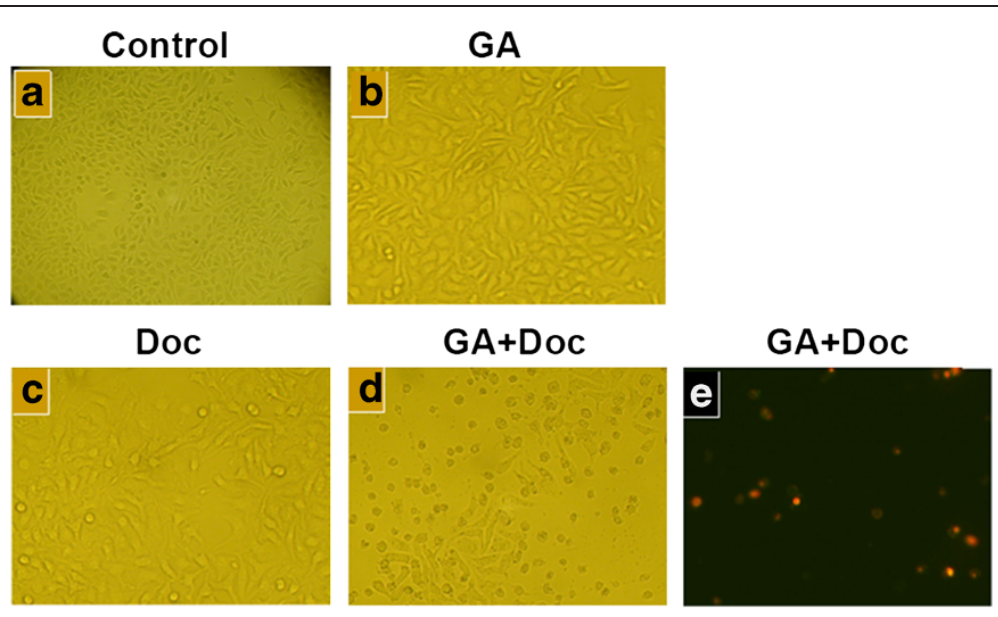

Figure 3 Induction of MKN-28 cell death by GA and Doc. After $48 \mathrm{~h}$ incubation with GA $(0.25 \mu \mathrm{M})$ (b), Doc $(0.625 \mu \mathrm{M})(\mathbf{c})$ or GA (0.25 $\mu \mathrm{M})$ plus Doc $(0.625 \mu \mathrm{M})(\mathbf{d})$, morphological changes in MKN-28 cells were observed under light microscopy. Cells without any treatments were used as controls (a). (e) Annexin-V and PI double staining for cells from panel d). Samples were visualized under a fluorescent microscope. Annexin-Vlabeled cells with green fluorescence were recognized as early apoptotic cells; annexin-V and PI double-labeled cells with orange fluorescence were recognized as late apoptotic or necrotic cells. 
pronounced synergistic effect for the combination therapy. These results are summarized in Table 2, which indicates for each combination, a computer-calculated CI for $20 \%, 40 \%, 60 \%$ and $80 \%$ cytotoxicity $(\mathrm{Fa}=0.2,0.4$, $0.6,0.8$, respectively).

\section{Combination treatment enhances apoptotic cell death}

Based on the results obtained with the MTT assay and the middle-effect principle, the lower concentration of GA plus Doc could also induce remarkable growth suppression in gastrointestinal cancer cells. Hence, $0.25 \mu \mathrm{M}$ GA and $0.625 \mu \mathrm{M}$ Doc were administered for evaluation of apoptotic cell death in MKN-28 cells. Forty-eight hours after drug incubation, the morphological alterations of MKN-28 cells were observed using light microscopy. As shown by Figure 3, increased cell loss was found after GA $(0.25 \mu \mathrm{M})$ plus Doc $(0.625 \mu \mathrm{M})$ treatment. Similar with the results obtained from MKN-28 cells, Annexin-V and PI double staining of the other three cell lines all revealed that the combination treatment induced apoptotic cell death (data

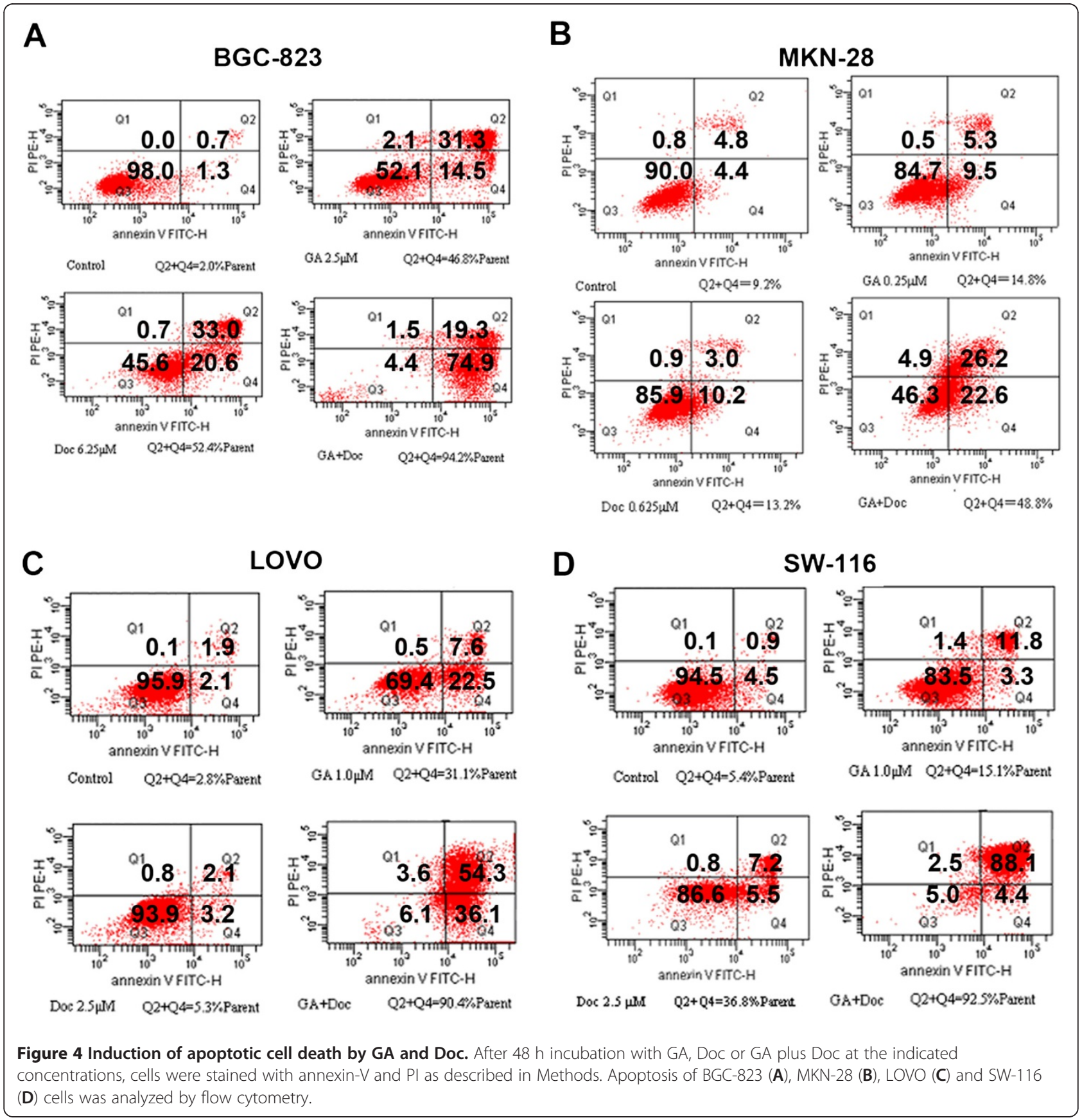


not shown). Apoptosis of BGC-823, MKN-28, LOVO and SW-116 cells after combination treatment was further determined by flow cytometry. Consistent with previous observations, the proportion of apoptotic cells (annexin $\mathrm{V}^{+}$ $\mathrm{PI}^{+/-}$) was markedly increased by application of GA plus Doc in all four cell lines as compared with GA or Doc treatment alone (Figure 4). These results indicate that the combination treatment enhanced apoptotic cell death in gastrointestinal cancer cells.

\section{GA downregulates the expression of genes involved in docetaxel sensitivity}

Accumulating evidence indicates that various cellular proteins that are associated with microtubules can determine the sensitivity of cancer cells to microtubuletargeting agents and play a role in tumor cell resistance to these agents [13]. To explore the potential mechanism by which GA plus Doc induces apoptotic cell death, the expression of genes involved in Doc sensitivity that regulate microtubules, such as class III $\beta$-tubulin $(\beta$-tubulin III) and tau, were evaluated by qRT-PCR. It should be noted that $48 \mathrm{~h}$ of $\mathrm{GA}(0.25 \mu \mathrm{M})$ incubation markedly down-regulated the expression of $\beta$-tubulin III and tau (Figure 5). Moreover, the expression of survivin, which is recognized as an apoptosis inhibitor, [14] was also suppressed by GA administration (Figure 5). These results imply that $\beta$-tubulin III, tau and survivin might be involved in the GA-mediated anti-tumor effect.

\section{Discussion}

Gastrointestinal cancer is the most commonly diagnosed cancer worldwide, exhibiting a high annual mortality [13]. Chemotherapy has been recognized as the treatment of choice for patients with gastric or colorectal cancer, either alone or in combination with surgery with or without radiotherapy $[15,16]$. Various chemotherapeutic agents have been used for clinical treatment of patients, including mitomycin $\mathrm{C}$, cisplatin, doxorubicin, 5-fluorouracil (5-FU), paclitaxel and Doc [17]. It has been reported that the response rates range from $10 \%$ to $30 \%$ for single-agent therapy and $30 \%$ to $60 \%$ for combination therapy [17]. However, the overall outcome remains poor due to local or distant recurrences, drug resistance or side effects derived from the various drugs. Accumulating evidence suggests that certain natural phytochemical compounds used in conjunction with chemotherapeutic agents could enhance therapeutic efficacy by sensitizing cells to treatment [18].

Doc, which is widely used for the treatment of several human malignancies, has been reported to be active against gastric cancer $[8,9]$. However, administration of Doc alone often leads to undesirable side effects and drug resistance $[9,16]$. The development of new taxane anticancer agents with fewer side effects, superior pharmacological properties and improved activity against drug-resistant human cancers seems to be a critical need for treatment of gastric cancer [19]. Moreover, Doc-containing chemotherapy has gradually developed with the aim of improving overall response rate and survival [10].

GA, the primary active component of gamboge, has been reported to induce apoptotic cell death of human gastric cancer SGC-7901 and BGC-823 cells [20,21]. Treatment of Doc resistant BGC-823/Doc cells with GA led to a dramatic increase in Doc-induced cytotoxicity. Cell cycle analysis further indicated that GA treatment potentiated Doc-induced G2/M cell cycle arrest. Moreover, GA singly or in combination with Doc significantly downregulated the mRNA expression of survivin [11]. In addition, combined treatment of GA and 5-FU caused significant growth inhibition of BGC-823 human tumor xenografts in vivo [22] Our previous study demonstrated that GA reversed Doc resistance in BGC-823/Doc gastric cancer cells [11]. In the present study, the combined application of GA and Doc exhibited synergistic and efficient anti-tumor effects in the human gastric cancer cell lines MKN-28 and BGC-823, and colorectal cancer cell lines LOVO and SW-116 (Figures 1 and 2), suggesting that GA may enhance the anti-cancer effects of Doc in both gastric and colorectal cancers. In addition, combination therapy suppressed the proliferation of cancer cells in a dose-dependent manner (Figure 1). Furthermore, the colorectal cancer cell line LOVO exhibited the lowest

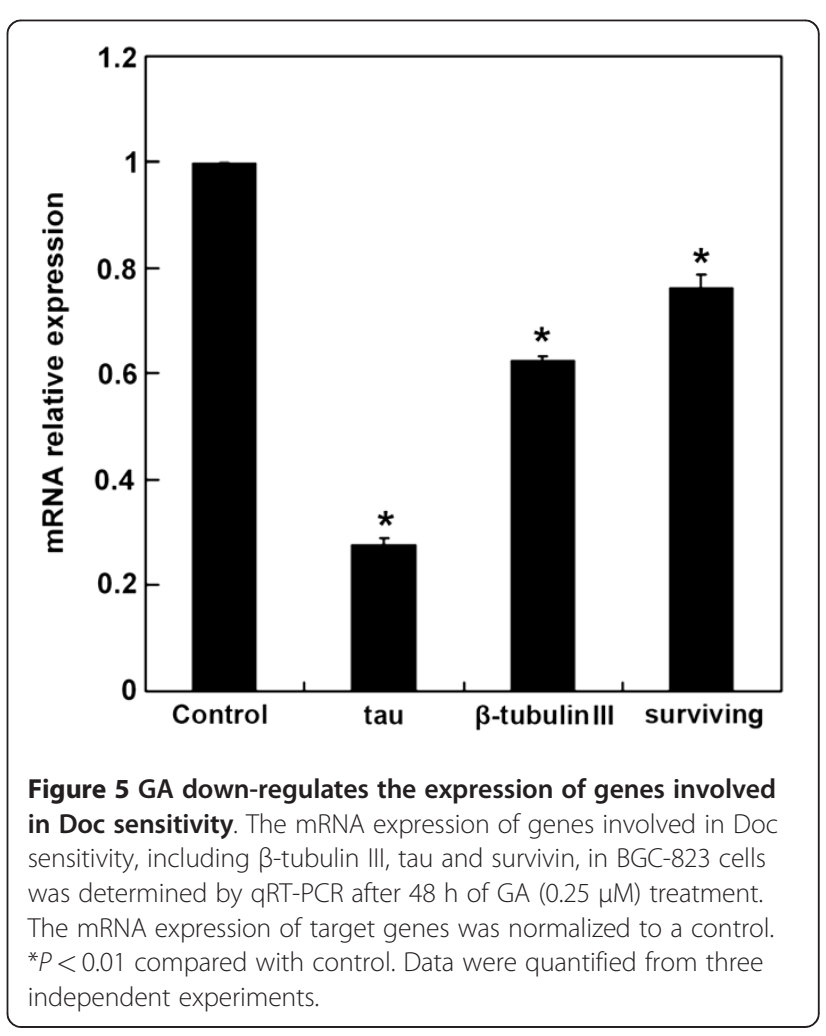


$\mathrm{IC}_{50}$ following GA administration, followed by the highly differentiated human gastric cancer cell MKN-28. With regard to Doc treatment, SW-116 cells were the most sensitive cell line followed by BGC-823 cells. However, combined administration of GA and Doc provided a synergistic effect on growth suppression of MKN-28 cells. Based on these observations, we hypothesize that growth of highly differentiated tumor cells, such as MKN-28 cells, could be synergistically suppressed by GA and Doc treatment. These observations are in accordance with previous reports showing that the combined application of 5-FU and GA had a stronger anti-gastric cancer effect in BGC-823 cells than that of 5-FU or GA alone [22].

Survivin, a novel inhibitor of apoptosis (IAP), has been reported to be associated with apoptosis, proliferation and angiogenesis during human colorectal tumorigenesis [23]. Moreover, survivin is also involved in tumor cell resistance to certain anti-cancer agents [24]. Our previous study demonstrated that GA reversed Doc resistance in BGC-823/Doc gastric cancer cells by down-regulating the expression of survivin,[11] implying that GA may promote the anti-tumor effect of Doc through mediation of apoptotic cell death. Here, we found that combination therapy significantly enhanced cell apoptosis (Figures 3 and 4). In addition, GA reduced the mRNA expression of survivin, suggesting that apoptosis might be involved in the GA-mediated anti-tumor effect.

Microtubule-associated proteins, such as $\beta$-tubulin III and tau, are known as essential predictive markers for the sensitivity of taxane-based chemotherapy in several human cancers $[25,26]$. Reduced expression of microtubule-associated proteins is linked to better outcomes for taxane therapy. Doc binds to $\beta$-tubulin, which is one of the major components of microtubules, and exerts its growth-inhibitory effects through G2/M cell cycle arrest. This then induces mitochondrial dysfunction and cell apoptosis. In the present study, GA treatment markedly decreased the mRNA expression of $\beta$-tubulin III and tau, suggesting these two genes were also involved in the GA-induced anti-tumor effect.

In summary, our present study demonstrates that GA and Doc combination treatment induced a synergistic, anti-tumor effect in gastric and colorectal cancer cells. Future studies will be focused on exploring the mechanism of the interaction of GA and Doc in gastrointestinal cancer cells and investigating the combination therapy in vivo using animal models.

\section{Competing interests}

The authors declare that they have no competing interests.

\section{Acknowledgements}

This work was supported by a grant from the key program of Nanjing Medical Science and Technique Development Foundation (Grant No. ZKX10011).

\section{Author details}

'The Comprehensive Cancer Center of Drum Tower Hospital, Medical School of Nanjing University \& Clinical Cancer Institute of Nanjing University, Nanjing 210008, China. ${ }^{2}$ Department of Laboratory, Drum Tower Hospital, Medical School of Nanjing University, Nanjing 210008, China. ${ }^{3}$ Medical School of Nanjing University, Nanjing 210008, China.

\section{Authors' contributions}

Zhengyun Zou designed the study protocol, performed synergistic experiments and prepared the manuscript. Li Xie, Jia Wei, LixiaYu and Tingting Wang performed qRT-PCR. Xiaoping Qian performed statistical analysis. Junhao Chen analyzed apoptosis. Baorui Liu designed the study protocol together with the first author. All authors read and approved the final manuscript.

Received: 10 August 2011 Accepted: 30 April 2012

Published: 30 April 2012

\section{References}

1. Crew KD, Neugut Al: Epidemiology of gastric cancer. World J Gastroenterol 2006, 12(3):354-362.

2. Parkin DM, et al: Global cancer statistics, 2002. CA Cancer J Clin 2005, 55(2):74-108.

3. Kamangar F, Dores GM, Anderson WF: Patterns of cancer incidence, mortality, and prevalence across five continents: defining priorities to reduce cancer disparities in different geographic regions of the world. J Clin Oncol 2006, 24(14):2137-2150.

4. Janunger KG, Hafstrom L, Glimelius B: Chemotherapy in gastric cancer: a review and updated meta-analysis. Eur J Surg 2002, 168(11):597-608.

5. Ocvirk J, et al: Cetuximab plus FOLFOX6 or FOLFIRI in metastatic colorectal cancer: CECOG trial. World J Gastroenterol 2010, 16(25):3133-3143.

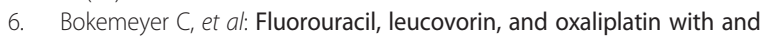
without cetuximab in the first-line treatment of metastatic colorectal cancer. J Clin Oncol 2009, 27(5):663-671.

7. Trudeau ME: Docetaxel: a review of its pharmacology and clinical activity. Can J Oncol 1996, 6(1):443-457.

8. Mavroudis $\mathrm{D}$, et al: Frontline treatment of advanced gastric cancer with docetaxel and granulocyte colony-stimulating factor (G-CSF): a phase II trial. Am J Clin Oncol 2000, 23(4):341-344.

9. Einzig Al, et al: Phase II trial of docetaxel (Taxotere) in patients with adenocarcinoma of the upper gastrointestinal tract previously untreated with cytotoxic chemotherapy: the Eastern Cooperative Oncology Group (ECOG) results of protocol E1293. Med Oncol 1996, 13(2):87-93.

10. Ridwelski $K$, et al: Combination chemotherapy with docetaxel and cisplatin for locally advanced and metastatic gastric cancer. Ann Oncol 2001, 12(1):47-51.

11. Wang T, et al: Gambogic acid, a potent inhibitor of survivin, reverses docetaxel resistance in gastric cancer cells. Cancer Lett 2008, 262(2):214-222.

12. Chou TC, Talalay P: Quantitative analysis of dose-effect relationships: the combined effects of multiple drugs or enzyme inhibitors. Adv Enzyme Regul 1984, 22:27-55.

13. Bhat KM, Setaluri V: Microtubule-associated proteins as targets in cancer chemotherapy. Clin Cancer Res 2007, 13(10):2849-2854.

14. Johnson ME, Howerth EW: Survivin: a bifunctional inhibitor of apoptosis protein. Vet Pathol 2004, 41(6):599-607.

15. Woll E, et al: Chemotherapy in gastric cancer. Anticancer Res 2008, 28(2):1213-1219.

16. Goyle S, Maraveyas A: Chemotherapy for colorectal cancer. Dig Surg 2005, 22(6):401-414

17. Sastre J, Garcia-Saenz JA, Diaz-Rubio E: Chemotherapy for gastric cancer. World J Gastroenterol 2006, 12(2):204-213.

18. Alisi A, Balsano C: Enhancing the efficacy of hepatocellular carcinoma chemotherapeutics with natural anticancer agents. Nutr Rev 2007, 65(12 Pt 1):550-553.

19. Geney R, Chen J, Ojima I: Recent advances in the new generation taxane anticancer agents. Med Chem 2005, 1(2):125-139.

20. Guo QL, et al: Gambogic acid inducing apoptosis in human gastric adenocarcinom SGC-7901 cells. Chin J Nat Med 2004, 2:107-110. 
21. Liu W, et al: Anticancer effect and apoptosis induction of gambogic acid in human gastric cancer line BGC-823. World I Gastroenterol 2005,

11(24):3655-3659.

22. Wang J, et al: Synergistic effect of 5-fluorouracil with gambogic acid on BGC-823 human gastric carcinoma. Toxicology 2009, 256(1-2):135-140.

23. Kawasaki $\mathrm{H}$, et al: Expression of survivin correlates with apoptosis, proliferation, and angiogenesis during human colorectal tumorigenesis. Cancer 2001, 91(11):2026-2032

24. Zaffaroni N, Daidone MG: Survivin expression and resistance to anticancer treatments: perspectives for new therapeutic interventions. Drug Resist Updat 2002, 5(2):65-72.

25. Urano $\mathrm{N}$, et al: Clinical significance of class III beta-tubulin expression and its predictive value for resistance to docetaxel-based chemotherapy in gastric cancer. Int J Oncol 2006, 28(2):375-381.

26. Rouzier $\mathrm{R}$, et al: Microtubule-associated protein tau: a marker of paclitaxel sensitivity in breast cancer. Proc Natl Acad Sci U S A 2005, 102(23):8315-8320

doi:10.1186/1472-6882-12-58

Cite this article as: Zou et al: Synergistic anti-proliferative effects of gambogic acid with docetaxel in gastrointestinal cancer cell lines. BMC Complementary and Alternative Medicine 2012 12:58.

\section{Submit your next manuscript to BioMed Central and take full advantage of:}

- Convenient online submission

- Thorough peer review

- No space constraints or color figure charges

- Immediate publication on acceptance

- Inclusion in PubMed, CAS, Scopus and Google Scholar

- Research which is freely available for redistribution 